\title{
Fast Road Sign Detection Using Hough Transform for Assisted Driving of Road Vehicles
}

\author{
Miguel Ángel García-Garrido, Miguel Ángel Sotelo, and Ernesto Martín-Gorostiza \\ Department of Electronics, University of Alcalá, Alcalá de Henares, Madrid, Spain \\ \{garrido, michael, ernesto \} @depeca.uah.es
}

\begin{abstract}
A system for real-time traffic sign detection is described in this paper. The system uses restricted Hough transform for circumferences in order to detect circular signs, and for straight lines for triangular ones. Some results obtained from a set of real road images captured under both normal and adverse weather conditions are presented as well in order to illustrate the robustness of the detection system. The average processing time is $30 \mathrm{~ms}$ per frame, what makes the system a good approach to work in real time conditions.
\end{abstract}

\section{Introduction}

Traffic sign detection and recognition has experimented increasing research interest in the last times. This is due to the importance of improving safety in road vehicles. Drivers sometimes miss signs because of distractions or lack of concentration. The two main causes of car-accidents in Spain are speed limit exceeding and distractions in general, so, aid to keeping the speed below the limit and avoiding distraction while driving are the two main targets in this work which is focussed on traffic sign detection for driving assistance. But the driver is kept in the control-loop, thus, our system will alert drivers, but will not actuate in order to control the vehicle. This can be done, for example, using acoustic warning if speed is over the limit, noticing the presence of a sign in a display, or by means of an audio signal (synthesized human voice) indicating the detection of a certain sign. Traffic-sign detection and recognition systems were born at the late 80 's, but it has not been until recent times that real timeperforming systems have been successfully achieved [1], [2], [3]. The most common method used for traffic signs detection is colour-segmentation. This method is based on the assumed fact that the wavelength coming from a sign, for instance the redcoloured edge of a speed-limit sign, does not change with changes in the intensity and the incidence-angle of the light onto the sign, if HSV or HIS spaces are used [4], [5]. The image of the camera is not, however, completely invariant under changes in the chromaticity of the received light, being these changes due to shades, adverse weather conditions, etc. Other authors use the information of scene-shapes for sign detection. Among these ones, some of them apply a template to an edge-image [1], [3]. The method consists in obtaining the distance-transform from an edge-image and doing a further matching with pre-selected templates, corresponding to those signals searched; these templates are hierarchically organized so that the number of comparisons is reduced, but this method implies nevertheless quite a high computational cost for a 
real time system. In other works, Hough transform is used [10], or else a varied version of it [2], [7]. In these works the information about symmetry-properties of the objects is used [8]. Barnes and Zelinsky [2] show that by applying this method the system is fast enough to work in real time, but only with circular signs, being only specifically tested for 40 and $60 \mathrm{~km}$ per hour- speed-limit signs, while Loy and Barnes [7] have used a similar technique for triangular, square, and octagonal signs, but not working in real time.

This work can be included with those that use information from the shape of the objects in the scene, in order to reduce the number of possible sign candidates. From the four types of signs existing in the Spanish driving code, prohibition, obligation, warning, and informative, the two first except for the stop one are circular signs. Nevertheless this one can be also considered as circular for detection issues. Sign detection has been performed by using the Hough transform for circumferences [11], but with certain restrictions that make it very efficient for the detection of this kind of signs. For warning signs, which are triangular, Hough transform for straight lines has been chosen, as a triangular sign is formed by three straight lines cutting each other under $60^{\circ}$ angles, in pairs. Informative signals are not considered in this work.

Finally, it must be noted that the system presented in this work focuses the searcharea only in a certain pre-selected zone of the image, that one holding a higher probability of finding a traffic sign inside.

\section{Contours Information}

The method used for edge detection is Canny method [9]; this method preserves contours, what is very important for detecting traffic signs using shape information, because they are usually closed contours, as can be seen in figure 1 .
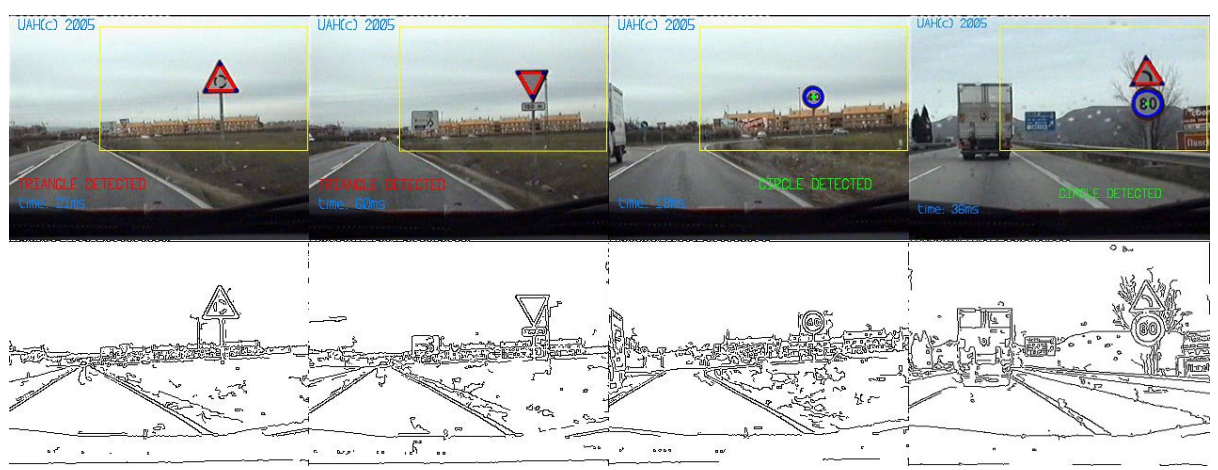

Fig. 1. Real images sequence, detected on the road, with the search area within each image outlined (square window), and Canny image used for contour-information search

Numerous implementations of edge-detection-systems based on Canny's idea have been developed. Canny described a method for generating a robust edge-detector. This method generates thin contours, which also avoids different contours from different objects joining together. Several tests, under different weather and 
illumination conditions have been taken, trying different threshold levels and being the results very similar in all cases. This shows that the value of Canny threshold levels is not critical for this application.

The contours obtained applying Canny method are codified using the 'chain code'. The contours are accepted if they are closed contours, or almost closed contours. They must also fulfil a certain aspect-ratio constraint, showing similar width and height. circular traffic signs, including stop one, as well as triangular ones, meet these restrictions with high probability. Hough transform is only applied to accepted contours after filtered with the aforementioned restrictions, so that the computational time is reduced.

\section{Hough Transform}

The classical Hough algorithm can be easily extended to find any curves in an image that can be expressed analytically in the form $f(x, p)=0$ [12]. Here, $x$ is a point in the domain of the image and $p$ is a parameter vector.

Hough transform for straight lines is applied in order to detect triangular signs. The aim is detecting three straight lines intersecting each other, forming a 60 degreesangle. It must be observed that, as long as the number of straight lines intersecting each other might be very large if the Hough transform was applied to the whole image, more than the actual triangles existing in the image would be detected. Using Hough transform neither the beginning nor the end of a straight line is known, as a straight line defined by this transform is expressed in terms of the polar parameters $\theta$ and $\rho$ :

$$
x \cos (\theta)+y \sin (\theta)=\rho
$$

In order to overcome this handicap in this work the strategy is to apply the Hough transform to every contour, one after the other. In this way, only those triangles existing actually in the image are detected, as shown in figure 2 , reducing the computational time too.
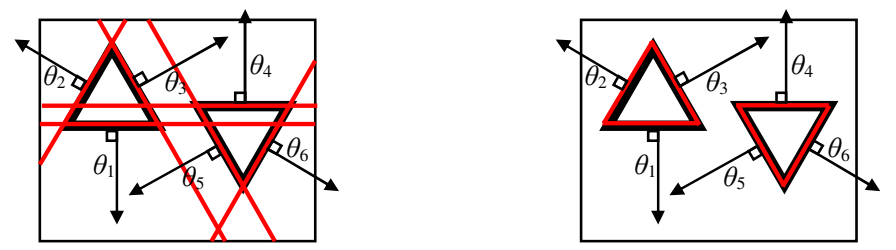

Fig. 2. Straight lines detected using Hough transform, applied to the whole image (left) applied to each contour, one by one (right)

Hough transform for circumferences is applied to detect circular signs, and the stop sign too. A circle in the xy-plane with center $(\chi, \psi)$ and radius $\rho$ can be expressed as:

$$
f(x, p)=(x-\chi)^{2}+(y-\psi)^{2}-\rho^{2}=0
$$

Where the parameter space, $\mathrm{p}=(\chi, \psi, \rho)$, must be quantized. The accumulator matrix ' $a$ ' is the representation of the quantized parameter space. For circumference detection 
the accumulator ' $a$ ' will be a three-dimensional matrix with all entries initially set to 0 . The entry a $\left(\chi_{\mathrm{r}}, \psi_{\mathrm{s}}, \rho_{\mathrm{t}}\right)$ is incremented by 1 for every feature point $\left(\mathrm{x}_{\mathrm{i}}, \mathrm{y}_{\mathrm{i}}\right)$ in the image-domain, contained in the circumference with centre $\left(\chi_{\mathrm{r}}, \psi_{\mathrm{s}}\right)$ and radius $\rho_{\mathrm{t}}$ as expressed in (3) where a precision margin $\varepsilon$ for the radius $\rho_{\mathrm{t}}$ is introduced to compensate quantization error when digitizing the image [13]:

$$
\mid\left(\chi_{\mathrm{r}}-\mathrm{x}_{\mathrm{i}}\right)^{2}+\left(\psi-\mathrm{y}_{\mathrm{i}}\right)^{2}-\rho_{\mathrm{t}}{ }^{2} \mathrm{<}<\varepsilon
$$

For circular-objects detection the same criteria are followed as in the case of straight lines. Hough transform is applied contour by contour, so that those contours corresponding to other shapes but not signs do not affect the detection of the latter ones, as shown in figure 3 .
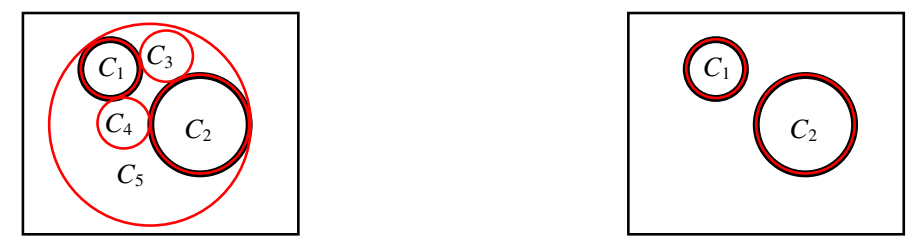

Fig. 3. Circumferences detected using Hough transform for circumferences, applied to the whole image (left) applied to each contour, one by one (right)

One important feature of a circular sign is that its centre and its centroid are in fact the same point. Making use of this property the centre is sought in a search-scope near the centroid. All these considerations make detection time to be very short, making the system able to work at a processing-speed between 5 and 50 frames per second, depending on the number of signs detected.

\section{Results}

The system works with one only camera mounted on the windscreen of the car, as shown in figure 4. Several tests have been conducted, placing the camera in different positions on the windscreen, and it has been concluded that the placement of the camera is not decisive, but orientation is, thus affecting the quality of detection. The best arrangement is to place the camera pointing towards the same direction and sense of the car so that signs are seen orthogonally to the motion-direction and thus suffering the least possible distortion. Should a circular sign be captured nonorthogonally by the camera, it would be seen as an ellipse in the image and would not be detected. However, Hough transform can be extended to ellipses, but it would be necessary to add two new parameters in the parameter-space with respect to the transform for circumference used in this work. This technique has been tested in fact, and it was noticed that the average processing time was 2 seconds, so the system could not operate in real time. It is important to realize that detecting circumferences but not ellipses is in fact a simplification of the method, but it does not imply a poorer performance at all. On the contrary, an elliptical shape in the image captured, if it 
happened to correspond to a sign, would be placed with high probability in another road with other direction, for instance in a crossroads. So, only those signs detected as circular are placed in our road in the egomotion direction.
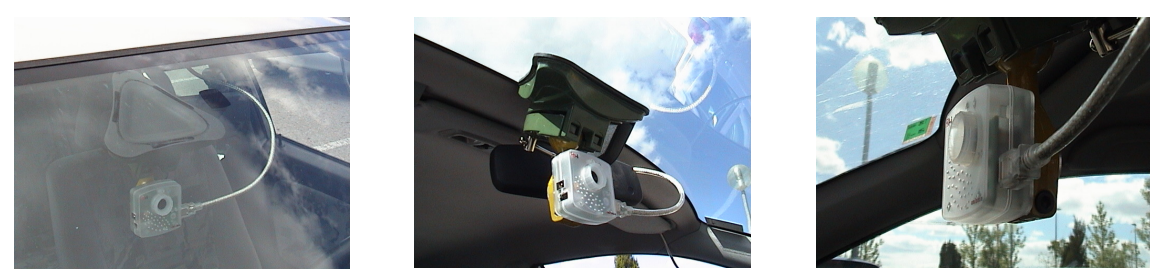

Fig. 4. One camera mounted on the windscreen of the car

The system has been empirically tested under severe adverse weather conditions, as it is depicted in figure 5, and the successful-detection ratio has not been affected. For every test made, the successfully-detected-sign percentage has been, over $99 \%$ with an average processing time of $30 \mathrm{~ms}$ per frame.

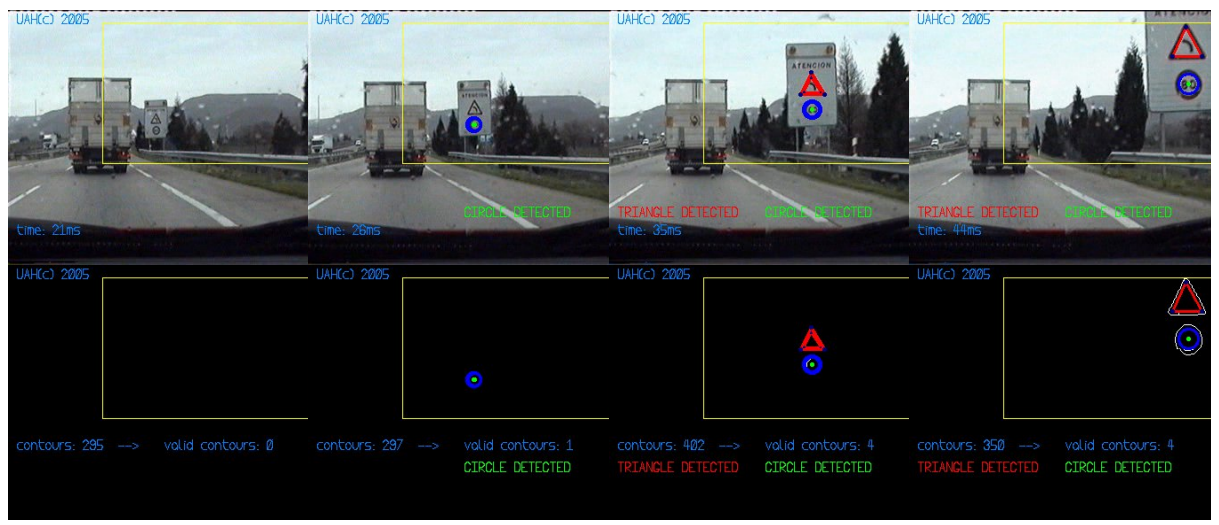

Fig. 5. Sequence of real road images under adverse weather conditions where speed limit and triangular sign are detected

\section{Conclusions}

A real time-algorithm for traffic signs detection has been shown. The algorithm is able to detect any kind of signs but the informative ones, using a similar technique for all of them, making the algorithm very robust. Besides, the position of the camera in the car is not critical and it is fast enough so as to work in real time without any problems. Another important feature is that it shows the same good performance under adverse weather conditions, for example in a rainy day.

As future work, a kalman filter to make a continuous and soft tracking of detected signs until they are not present in the field of view will be added. By doing so, 
continuous detection of the same sign would be avoided. Another approach to be done is to implement the classification or recognition stage, which will be done with a neural network, the most widely used method for this purpose.

\section{Acknowledgments}

This work has been funded by Research Projects CICYT DPI2002-04064-05-04 (Ministerio de Educación y Ciencia) and FOM2002-002 (Ministerio de Fomento, Spain).

\section{References}

1. Gavrila, D.M.; Franke, U.; Wohler, C.; Gorzig, S. "Real time vision for intelligent vehicles," Instrumentation \& Measurement Magazine, IEEE Volume 4, Issue 2, June 2001 Page(s): 22-27.

2. Barnes, N.; Zelinsky, A. "Real-time radial symmetry for speed sign detection," Intelligent Vehicles Symposium, 2004 IEEE, 14-17 June 2004 Page(s):566 - 571.

3. Gavrila, D.M.; Philomin, V. "Real-time object detection for "smart" vehicles," Computer Vision, 1999. The Proceedings of the Seventh IEEE International Conference on, Volume 1, 20-27 Sept. 1999 Page(s):87 - 93 vol.1.

4. Priese L., Rehrmann V., Schian R., Lakmann R.,"Traffic Sign Recognition Based on Color Image," Evaluation IEEE Intelligent Vehicles Symposium'93, Tokyo, 1993.

5. De Micheli, E.; Prevete, R.; Piccioli, G.; Campani, M., "Color cues for traffic scene analysis," Intelligent Vehicles '95 Symposium., Proceedings of the25-26 Sept. 1995 Page(s): 466 - 471.

6. C. Y. Fang, C. S. Fuh, S. W. Chen, and P. S. Yen, "A road sign recognition system based on dynamic visual model" in Proc IEEE Conf. on Computer Vision and Pattern Recognition, vol. 1, 2003, pp. 750-755.

7. Loy, G.; Barnes, N.;"Fast shape-based road sign detection for a driver assistance system," Intelligent Robots and Systems, 2004. (IROS 2004). Proceedings. 2004 IEEE/RSJ International Conference on, Volume 1, 28 Sept.-2 Oct. 2004 Page(s):70 - 75 vol.1

8. Loy, G.; Zelinsky, A.;"Fast radial symmetry for detecting points of interest," Pattern Analysis and Machine Intelligence, IEEE Transactions on, Volume 25, Issue 8, Aug. 2003 Page(s):959 - 973

9. J. Canny. "A Computional approach to Edge-Detection," IEEE Transactions on pattern Analysis and Machine Intelligence, vol 8, pp. 679-700, 1986.

10. P. Hough, "Method and means for recognizing complex patterns," Dec. 18 1962. U.S. Patent 3,069,654

11. R. Duda and P. Hart, "Use of the Hough transform to detect lines and curves in pictures," Communications of the ACM, vol. 15, no. 1, pp. 11-15, 1972.

12. D. Ballard, "Generalizing the Hough transform to detect arbitrary shapes," Pattern Recognition, vol. 13, no. 2, pp. 111-122, 1981.

13. S. D. Shapiro, "Properties of transforms for the detection of curves in noisy image," Computer Graphics and Image Processing, vol. 8, pp. 219-236, 1978. 